\title{
First $L$ band detection of hot exozodiacal dust with VLTI/MATISSE
}

\author{
Florian Kirchschlager ${ }^{\circledR},{ }^{1 \star}$ Steve Ertel, ${ }^{2,3}$ Sebastian Wolf, ${ }^{4}$ Alexis Matter ${ }^{5}$ and Alexander V. Krivov ${ }^{6}$ \\ ${ }^{1}$ Department of Physics and Astronomy, University College London, Gower Street, London WC1E 6BT, UK \\ ${ }^{2}$ Large Binocular Telescope Observatory, 933 North Cherry Avenue, Tucson, AZ 85721, USA \\ ${ }^{3}$ Steward Observatory, Department of Astronomy, University of Arizona, 993 N. Cherry Ave, Tucson, AZ 85721, USA \\ ${ }^{4}$ Institute of Theoretical Physics and Astrophysics, Kiel University, Leibnizstraße 15, D-24118 Kiel, Germany \\ ${ }^{5}$ Laboratoire Lagrange, Observatoire de la Côte d'Azur, CNRS, Université Côte d'Azur, Boulevard de l'Observatoire, CS 34229, F-06304 Nice Cedex 4, France \\ ${ }^{6}$ Astrophysical Institute and University Observatory, Friedrich Schiller University Jena, Schillergäßchen 2-3, D-07745 Jena, Germany
}

Accepted 2020 September 4. Received 2020 September 4; in original form 2020 July 14

\begin{abstract}
For the first time, we observed the emission of hot exozodiacal dust in $L$ band. We used the new instrument MATISSE at the Very Large Telescope Interferometer to detect the hot dust around $\kappa$ Tuc with a significance of $3 \sigma$ to $6 \sigma$ at wavelengths between 3.37 and $3.85 \mu \mathrm{m}$ and a dust-to-star flux ratio of 5 to 7 per cent. We modelled the spectral energy distribution based on the new $L$ band data alone and in combination with $H$ band data published previously. In all cases we find $0.58 \mu \mathrm{m}$ grains of amorphous carbon to fit the $\kappa$ Tuc observations the best, however, also nanometre or micrometre grains and other carbons or silicates reproduce the observations well. Since the $H$ band data revealed a temporal variability, while our $L$ band data were taken at a different epoch, we combine them in different ways. Depending on the approach, the best fits are obtained for a narrow dust ring at a stellar distance in the 0.1-029 au range and thus with a temperature between 940 and $1430 \mathrm{~K}$. Within the $1 \sigma$ uncertainty dust location and temperature are confined to $0.032-1.18$ au and 600-2000 K.
\end{abstract}

Key words: techniques: interferometric-zodiacal dust-infrared: planetary systems.

\section{INTRODUCTION}

Since the first detection of hot exozodiacal dust ('hot exozodi') around Vega (Absil et al. 2006), about two dozens hot exozodis have been discovered using optical long baseline interferometry (Di Folco et al. 2007; Absil et al. 2008, 2009, 2013; Defrère et al. 2011, 2012; Ertel et al. 2014, 2016; Nuñez et al. 2017). Presumably accumulating at or close to the sublimation radius, the dust is heated to high temperatures and its emission peaks in the near-infrared (NIR) to mid-infrared (MIR). The detected dust-to-star flux ratios in NIR are at a level of a few per cent or even lower (e.g. Ertel et al. 2014). Thus, high precision (contrast) and high angular resolution $(\sim 0.01$ as) are required to observe hot exozodis.

The existence of hot exozodis raises questions as dust located at stellar distances of only $\sim 0.01-1$ au would be removed by radiative forces on time-scales of a few years. To be detectable around $\sim 20$ per cent of main-sequence stars of all spectral types from A to $\mathrm{K}$ at all ages (Ertel et al. 2014), the dust has to be continuously replenished or to be trapped in the stellar vicinity for long times, yet the eliciting mechanism has still to be identified (van Lieshout et al. 2014; Rieke, Gáspár \& Ballering 2016; Kral et al. 2017; Kimura et al. 2020; Pearce, Krivov \& Booth 2020).

Studies of hot exozodis offer a way to better understand the inner regions of extrasolar planetary systems. In addition, exozodis could help to trace the invisible sources of the dust and the putative planets at larger distances, and hence reveal the architecture of the planetary systems. On the other hand, the possible presence of small grains is a potential problem for the detection of terrestrial planets in the habitable zone (e.g. Agol 2007; Beckwith 2008).

So far, hot exozodis have only been observed in $H$ or $K$ band, thus grain sizes and grain compositions could not be constrained sufficiently. Previous modelling of the spectral energy distribution (SED) pointed towards nano- to sub-micrometre sized grains of carbonaceous material, however, larger grains could not be ruled out completely (Kirchschlager et al. 2017). $N$ band emission from more temperate (warm) dust near habitable zones has been detected (Millan-Gabet et al. 2011; Mennesson et al. 2014; Ertel et al. 2018b, 2020) but observations at intermediate wavelengths $(1.6 \mu \mathrm{m} \lesssim \lambda \lesssim$ $10 \mu \mathrm{m})$ are required to study the potential connection of warm and hot dust.

The Multi AperTure mid-Infrared SpectroScopic Experiment (MATISSE; Lopez et al. 2014) is a second-generation instrument at the Very Large Telescope Interferometer (VLTI), available since 2019. With a spatial resolution of a few mas and operating in $L$, $M$, and $N$ band, MATISSE offers critical capabilities for the study of hot exozodis. In particular, MATISSE will be able to confine the dust properties of hot exozodis (Ertel et al. 2018a; Kirchschlager et al. 2018).

$\kappa$ Tuc (HD 7788) is an F6 IV-V star located in the constellation Tucana at a distance of (21.0 \pm 0.3$)$ pc (Gaia Collaboration 2018) with an effective temperature of $6474 \mathrm{~K}$, stellar mass $\sim 1.35 \mathrm{M}_{\odot}$, and an age of $\sim 2$ Gyr (Ammler-von Eiff \& Reiners 2012; Fuhrmann et al. 2017; Tokovinin 2020). Significant hot emission was detected in 2012 and 2014 around $\kappa$ Tuc (Ertel et al. 2014). However, no 
Table 1. Observations of $\kappa$ Tuc (HD 7788) with VLTI/MATISSE [Program No. 0103.C-0725(A); PI: F. Kirchschlager].

\begin{tabular}{lcccc}
\hline ID & Date & Configuration & Seeing & Calib $^{a}$ \\
\hline A & $2019 / 07 / 09$ & K0-G2-D0-J3 & 0.8 as & 1,2 \\
B & $2019 / 07 / 11$ & K0-G2-D0-J3 & 0.8 as & 3,4 \\
\hline
\end{tabular}

${ }^{a}$ Calibrator stars correspond to (1) HD 3750 (K1III); (2) HD 8094 (K4III); (3) HD 4138 (K4III); and (4) HD 8315 (K0III).

significant excess was detected in 2013, making $\kappa$ Tuc the first hot exozodi candidate for significant NIR variability (Ertel et al. 2016).

In this letter, we present observations in $L$ band of the hot exozodi around $\kappa$ Tuc (Section 2) and a modelling of the observed visibilities and SED with the focus to constrain the dust properties in the circumstellar environment (Sections 3 and 4). We discuss the results in Section 5.

\section{OBSERVATIONS}

Interferometric $L M$ and $N$ band data were obtained on 2019 July 9 and 11 using the instrument MATISSE (Lopez et al. 2014) on the VLTI (Table 1). The Auxiliary Telescopes (ATs) were arranged in medium configuration and the New Adaptive Optics Module for Interferometry (NAOMI; Woillez et al. 2019) was used. We simultaneously obtained visibility measurements on six baselines (baseline lengths between $B=30$ and $95 \mathrm{~m}$ ). The observations were carried out in LOW spectral resolution $(R \sim 30)$. In $L M$ band, the fringes were dispersed over 64 spectral pixels between 3.28 and $4.57 \mu \mathrm{m}$, which correspond to about 13 true spectral channels (spectral channels are sampled over 5 spectral pixels on the $L M$ band detector). Our measurements thus cover a significant part of the $L$ band and the very beginning of the $M$ band. In $N$ band, $\kappa$ Tuc $(N \sim 1.5 \mathrm{Jy})$ is too faint for visibility measurements with MATISSE (to date sensitivity limit $\sim 15-20 \mathrm{Jy}$; see MATISSE ESO webpage). Even though $N$ band interferometric data were acquired simultaneously to the $L M$ band data, the independent step of $N$ band photometric measurements was thus skipped. No $N$ band visibility could be computed and we discarded the $N$ band data. In this work, our focus is on the $L M$ band data only.

Observations of $\kappa$ Tuc were framed by observations of two reference stars to calibrate the instrumental contribution (CALSCI-CAL sequence per night). Calibrators were chosen from the SearchCal tool (Chelli et al. 2016) to be regular main-sequence stars similar in magnitude and position of $\kappa$ Tuc and with a stellar angular diameter as low as possible.

The MATISSE data were reduced and calibrated with the help of SUV, the VLTI user support service of the JMMC. ${ }^{1}$ A specific aspect of MATISSE is the presence of two beam commuting devices (BCDs) that provide four independent beam configurations to calibrate out the effect of instrumental defects (Lopez et al. 2014): ININ, IN-OUT, OUT-IN, and OUT-OUT. A basic observation cycle in $L M$ band thus consists of four 1-min interferometric + photometric exposures, each of them being associated with one BCD position. Here, we focus on the absolute visibilities $V$ which requires to properly examine the four exposures for their visibility accuracy. For the observation on July 9, it turned out that the OUT-OUT exposure had to be discarded as the corresponding visibilities are inconsistent

\footnotetext{
${ }^{1}$ Website: http://www.jmmc.fr/suv.htm. Here, the last version of the MATISSE pipeline was used which is publicly available at http://www.eso.org/ sci/software/pipelines/matisse/.
}

(within $3 \sigma$ ) with the visibilities of the three other exposures, for most of the baselines. For the same reason, we discarded the IN-IN exposure of the July 11 observation.

Besides the 'short-term' errors of the pure fundamental source noise, thermal background and detector readout noise, broad-band errors on the photometry arise due to variations of the interferometric transfer function as well as due to an imperfect subtraction of the thermal background, which affect the visibility on a time-scale of $\sim$ min. The transfer function shows variations of less than 2 per cent on average, which is thus not a limiting factor for the visibility accuracy. We take the mean of the three exposures as final visibility for each spectral pixel and their 'long-term' error is estimated by the standard deviation of the three exposures.

Hence, the data set comprises 2 (observations) $\times 6$ (baselines) $=$ 12 independent visibility measurements for each wavelength. The final calibrated data set of two exemplary wavelengths plus the closure phase are shown in Fig. 1.

\section{ANALYSIS OF THE MATISSE DATA}

When we compare the calibrated data to the expected visibility of the stellar photosphere a visibility deficit is revealed. A stellar companion can be rejected as a source of this deficit as the closure phases are close to zero (Fig. 1), in agreement with measurements by Marion et al. (2014). Therefore, the visibility deficit must be caused by circumstellar emission in the field of view (FOV). We follow a two-step approach to determine the excess and the properties of the circumstellar material. In the first step (this section), the calibrated visibilities $V$ are fitted by a model consisting of a limb-darkened photosphere surrounded by a uniform disc emission filling the entire FOV of MATISSE. In the second step (Section 4), the fluxes derived in the first step are fitted to a disc model where the dust is arranged in a narrow ring and the grains' optical properties are considered. The two-step approach has been used in previous studies to constrain the dust properties (Absil et al. 2006, 2009; Di Folco et al. 2007; Absil et al. 2008; Defrère et al. 2011; Lebreton et al. 2013; Kirchschlager et al. 2017) and we will demonstrate at the end of Section 4 that the visibilities of the disc model are consistent to the MATISSE data which justifies this approach.

Using the flux ratio $f$ between the integrated circumstellar and the stellar photospheric emission, the combined visibility with contributions from the bare photosphere and from the circumstellar emission is (Di Folco et al. 2007)

$V_{(\star+\mathrm{CSE})}(B)=(1-f) V_{\star}(B)+f V_{\mathrm{CSE}}(B)$,

where

$V_{\star}(B)=\frac{6}{3-u_{\lambda}}\left(\left(1-u_{\lambda}\right) \frac{J_{1}(x)}{x}+u_{\lambda} \sqrt{\frac{\pi}{2}} \frac{J_{1.5}(x)}{x^{1.5}}\right)$

and $V_{\mathrm{CSE}}(B)=\exp \left(-\frac{x_{\mathrm{FOV}}^{2}}{4 \ln 2}\right)$

are the visibilities of a limb-darkening stellar model (Hanbury Brown et al. 1974) and of the circumstellar emission (symmetric Gaussian). Here, $x=\pi \Theta_{\star} B / \lambda, \Theta_{\star}=(0.739 \pm 0.011)$ mas is the stellar diameter (Ertel et al. 2014), $u_{\lambda}=0.22$ is the linear limb-darkening coefficient in $L M$ band (Claret, Diaz-Cordoves \& Gimenez 1995), $J_{1}(x)$ and $J_{1.5}(x)$ are Bessel functions, $x_{\mathrm{FOV}}=\pi \Theta_{\mathrm{FOV}} B / \lambda$, and $\Theta_{\mathrm{FOV}}=0.6$ as is the physical FOV in $L M$ band which is equivalent to $6.3 \mathrm{au}$ in radius at the distance of $\kappa$ Tuc. Under typical seeing conditions, the sensitivity along the FOV follows a Gaussian profile with a full width at half-maximum of the size of the FOV, FWHM $=0.6$ as. 

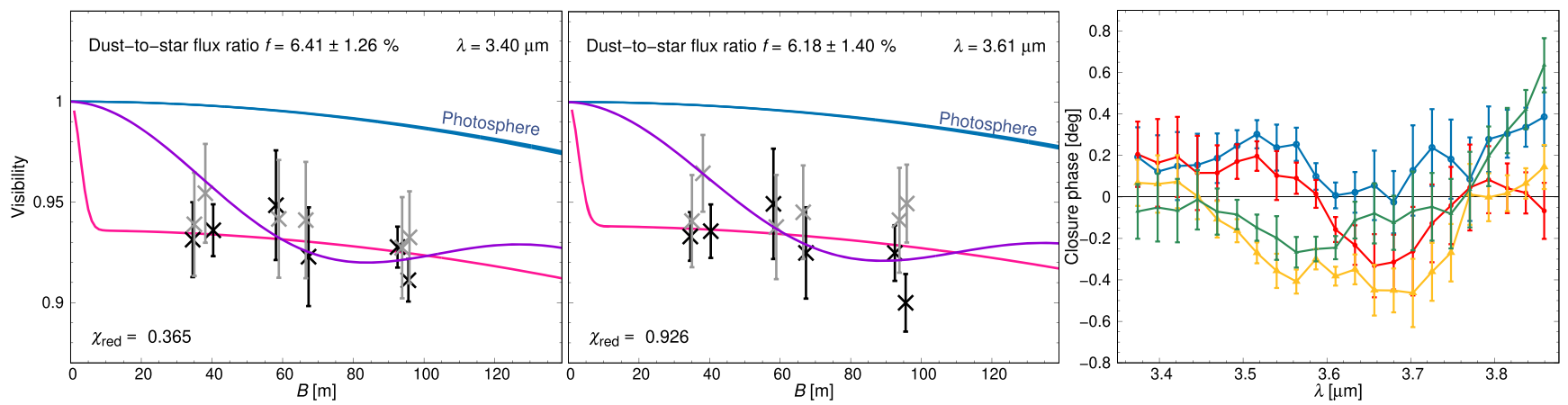

Figure 1. Left and Centre: Measured visibilities and related $1 \sigma$ errors (crosses and bars; observations on 2019 July 9 in black and on 2019 July 11 in grey) along with the expected visibility of the limb-darkened photosphere (blue line) as a function of projected baseline length $B$, for the wavelengths $\lambda=3.4$ and $3.61 \mu \mathrm{m}$. The thickness of the blue line corresponds to an adopted uncertainty of the stellar diameter. The best-fitting model is represented by the magenta line and corresponds to a uniform circumstellar emission (Gaussian profile) with a wavelength-dependent dust-to-star flux ratio $f$. The purple line represents the best fit of a disc modelling approach (Section 4). Right: Calibrated closure phases for the four triangles of the observation on July 9 . The 22 spectral elements range from 3.37 to $3.85 \mu \mathrm{m}$ and cover the region that show significant dust emission (see Section 3 for further details).

The dust-to-star flux ratio $f$ is the only free parameter of our model which has to be fitted for each wavelength. To find the bestfitting model, the calibrated MATISSE data of both observation nights are combined and fitted together (12 visibilities for each wavelength), the reduced $\chi^{2}$ is calculated as a function of $f$ and minimized. The statistical errors of $f$ are obtained by evaluating the probability distribution $p\left(\chi^{2}\right)=\exp \left(-\chi^{2}-\chi_{\text {best-fit }}^{2}\right)$ around the best-fitting model and by determining the corresponding confidence levels of $p$.

The visibility distribution of the best-fitting model has been overlaid on the plots of the calibrated data (Fig. 1, magenta line). The results of the fitting procedure are displayed as insets together with the $1 \sigma$ error of the flux ratio, $\pm \Delta f$, and the reduced $\chi^{2}$. The detection of the flux is significant when $f /|\Delta f| \geq 3$ which accounts for 22 wavelengths between 3.37 and $3.85 \mu \mathrm{m}$ which cover only the $L$ band. This is the first time ever hot exozodiacal dust has been detected in this waveband. The flux ratios of about 5-7 per cent are distinctly higher than the ratios known from $H$ and $K$ band for typical hot exozodis ( $\sim 1$ per cent; Absil et al. 2013; Ertel et al. 2014). On the other hand, no significant $(<3 \sigma)$ emission is detected at wavelengths above $3.85 \mu \mathrm{m}$. This is not because of larger uncertainties of the individual exposures but due to an increased dispersion of the data points of the exposures. The visibilities at wavelengths below $3.37 \mu \mathrm{m}$ increase to larger values and show either no significant deficit or no deficit at all.

Considering the emission of a stellar blackbody $\left(T_{\text {eff }}=6474 \mathrm{~K}\right)$ as a weighting factor for $f$, we calculated the flux of the significant circumstellar emission (Fig. 2). The fluxes show a steeply decreasing slope $F_{v} \propto \lambda^{-\alpha}$ with $\alpha=3.92_{-1.92}^{+2.68}$. Two features are visible in the spectrum at 3.5 and $3.75 \mu \mathrm{m}$. However, these features are not significant as can be seen by distinctly higher values for the reduced $\chi^{2}$ of 2.491 for $\lambda=3.5 \mu \mathrm{m}$ and 2.306 for $\lambda=3.75 \mu \mathrm{m}$ while it is about $1-1.5$ for neighbouring wavelengths, and are produced by a single visibility measurement on July 11 .

\section{MODELLING OF DUST PROPERTIES}

Besides the newly obtained MATISSE data, the hot exozodi of $\kappa$ Tuc has been observed three times in $H$ band using VLTI/PIONIER (Ertel et al. 2014, 2016). In 2012 and 2014, the circumstellar emission was significant with a flux ratio of $f=(1.43 \pm 0.17)$ per cent and

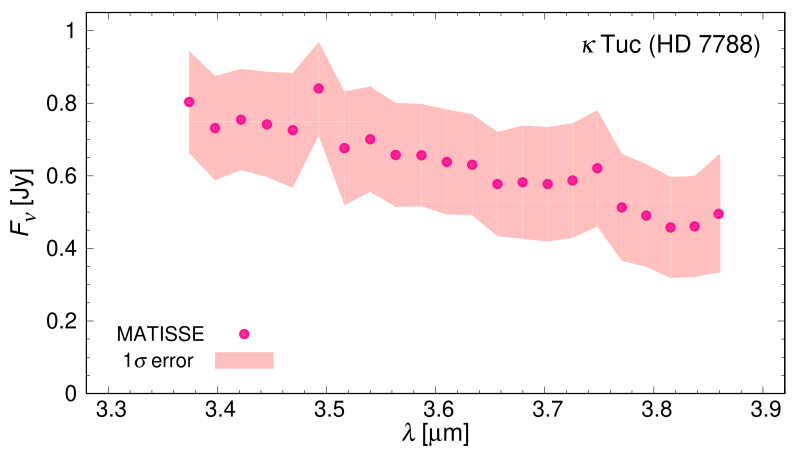

Figure 2. Spectral energy distribution (SED) of the circumstellar dust inferred from $L$ band observations with $>3 \sigma$ significance.

$(1.16 \pm 0.18)$ per cent, respectively, while the emission in 2013 was insignificant with $f=(0.07 \pm 0.16)$ per cent.

A blackbody fit to the SED is found wanting and we can exclude once for all a stellar companion as source of the circumstellar emission. Instead, we fit the SED by a disc model relying on the approach of Kirchschlager et al. (2017). In order to avoid parameter degeneracies, we keep the disc model as simple as possible and the number of free parameters as low as possible. Similar to the ring model of Absil et al. (2009) and Defrère et al. (2011), our model is represented by a narrow face-on ring with $R$ as inner radius, outer radius $1.1 R$, dust mass $M_{\text {dust }}$, and number density $n(r) \propto r^{-1}$. The ring is composed of compact spherical dust grains with radius $a$. Spherical grains with $2 a \lesssim \lambda \lesssim 10 a$ show in general strong interferences in the emission SED which can affect the fitting result. In order to reduce this effect we use a narrow size distribution with width $\Delta a=0.3 a$ around $a$, where the presence of different sizes causes the interferences to cancel each other out (e.g. Kirchschlager, Bertrang \& Flock 2019). The radial distance is varied in the range $R \in$ [0.03 au, $3 \mathrm{au}$, the grain size in the range $a \in[1 \mathrm{~nm}, 5 \mu \mathrm{m}]$, the dust mass in the range $M_{\text {dust }} \in\left[10^{-15} \mathrm{M}_{\oplus}, 10^{-3} \mathrm{M}_{\oplus}\right]$, and three different carbons and one silicate material are considered: Amorphous carbon (Rouleau \& Martin 1991), amorphous carbonaceous dust analogues (Jäger, Mutschke \& Henning 1998), crystalline graphite (Draine \& Lee 1984; Laor \& Draine 1993), and astronomical silicate (Weingartner \& Draine 2001). 
Table 2. Best-fitting results derived from SED modelling.

\begin{tabular}{lclcc}
\hline Appr. & $a(\mu \mathrm{m})$ & $R(\mathrm{au})$ & $T_{\text {dust }}(\mathrm{K})$ & $M_{\text {dust }}\left(10^{-9} \mathrm{M}_{\oplus}\right)$ \\
\hline (a) No NIR & 0.58 & 0.031 & 2300 & 0.54 \\
(b) 2012-2014 & 0.58 & 0.13 & 1260 & 3.11 \\
(c) 2012 and 2014 & 0.58 & 0.1 & 1430 & 2.0 \\
(d) 2013 & 0.58 & 0.29 & 940 & 10.4 \\
\hline
\end{tabular}

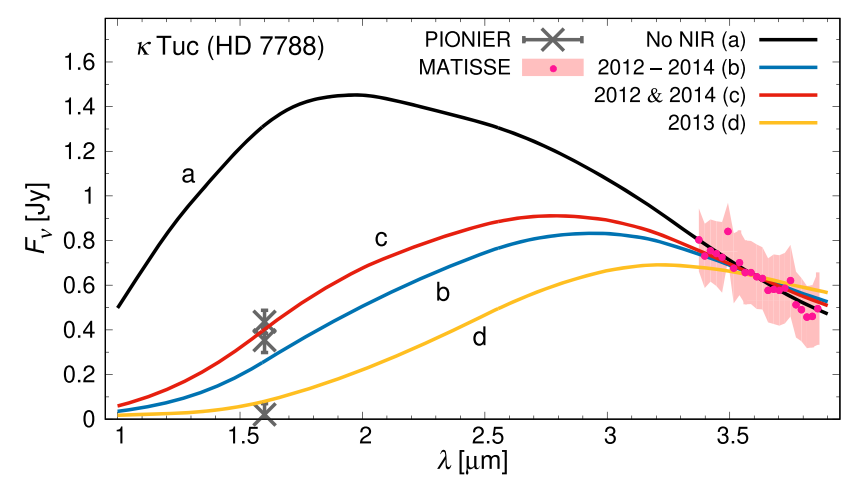

Figure 3. SED of the circumstellar dust emission around $\kappa$ Tuc composed of the $H$ band (grey) observations from 2012 to 2014 (Ertel et al. 2014, 2016) and $L$ band observations (magenta) from 2019 (this work). The solid lines represent the SEDs of the best-fitting models for amorphous carbon taking into account four different fitting approaches: (a) neglecting the NIR fluxes (black), (b) considering all $H$ band data (blue), (c) considering the 2012 and $2014 H$ band data (red), (d) considering $2013 H$ band data (yellow).

The grains' optical properties are calculated on the basis of Mie theory. Maps of single-scattering and re-emission are generated using an enhanced version of the tool DEBRIS (Ertel et al. 2011). Dust located within an inner working angle $\Theta=\lambda /(4 \times 100 \mathrm{~m})$ is assumed to be unresolved and its radiation is removed from the maps. The heterogeneous sensitivity along the FOV is taken into account by multiplying the simulated maps with a Gaussian function with an FWHM of 0.4 and 0.6 as for PIONIER and MATISSE, respectively. Finally, the SED is calculated from the synthesized maps and fitted to the observational data by minimizing the reduced $\chi^{2}$ and by varying the four free parameters $R, a, M_{\text {dust }}$, and the dust material. The corresponding confidence levels are again calculated by evaluating the probability function $p\left(\chi^{2}\right)=\exp \left(-\chi^{2}-\chi_{\text {best-fit }}^{2}\right)$ around the best-fitting model.

The main challenge is the $H$ band variability and the time that passed between the PIONIER and MATISSE observations, and we follow different fitting approaches to comply with. First (a), we solely fit the 22 significant $L$ band data points from MATISSE ( $\lambda \in$ $[3.37 \mu \mathrm{m}, 3.85 \mu \mathrm{m}])$. These data provide reliable information on the dust at the time of the MATISSE observations. Secondly, we assume that the three $H$ band measurements in 2012-2014 provide a probable range of the $H$ band excess during the MATISSE observations. We thus explore three scenarios when fitting MATISSE and PIONIER data together: (b) We include all three PIONIER measurements as this will give us the most likely result and a reasonable uncertainty on the dust parameters from the uncertainty of the PIONIER data. (c) We include only the two significant $H$ band excesses from 2012 and 2014. This provides a result where the $H$ band excess is on the high side of the observed range. (d) We include only the $H$ band nondetection from 2013, which gives the strongest upper limit available for the low side of $H$ band excesses observed. The results of our four fits are summarized in Table 2 and illustrated in Figs 3 and 4.
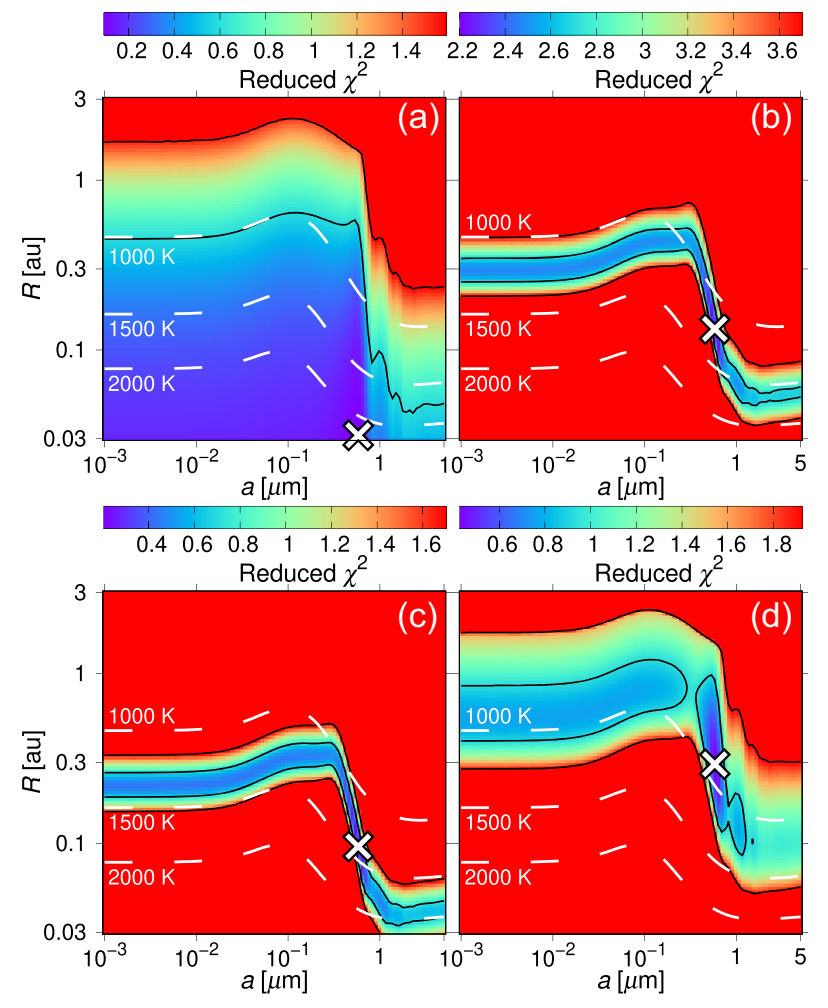

Figure 4. Reduced $\chi^{2}$ maps of the SED modelling of the hot exozodi of $\kappa$ Tuc as a function of grain size $a$ and disc radius $R$ for the four different fitting approaches (a)-(d). The white crosses indicate the best-fitting models and the black solid lines the $1 \sigma$ and $3 \sigma$ confidence levels. The dust temperatures $T_{\text {dust }}=1000,1500$, and $2000 \mathrm{~K}$ are shown as white dashed lines. The dust material is amorphous carbon.

For the first approach (a), all considered dust materials are able to reproduce the MIR slope, however, the disc radius and the grain size are weakly constrained with large $1 \sigma$ errors. For amorphous carbon the best-fitting model is composed of a disc radius $R=0.031$ au, grain size $a=0.58 \mu \mathrm{m}$, mass $M_{\text {dust }}=0.54 \times 10^{-9} \mathrm{M}_{\oplus}$, and the temperature is $2300 \mathrm{~K}$.

For the second approach (b), we find amorphous carbon to be the best-fitting material, which is able to reproduce the NIR and MIR fluxes and the MIR slope. The radiation is dominated by thermal re-emission which agrees with a detected lack of scattered radiation in polarization observations (Marshall et al. 2016). The best result $\left(\chi^{2}=2.199\right)$ is obtained for the grain size $a=0.58 \mu \mathrm{m}$, disc radius $R=0.13 \mathrm{au}$, mass $M_{\text {dust }}=3.11 \times 10^{-9} \mathrm{M}_{\oplus}$, and the dust temperature amounts to $T_{\text {dust }} \sim 1260 \mathrm{~K}$. However, within the $1 \sigma$ confidence level, the grain size is not constrained by our modelling and both nanometre-sized grains and those as large as a few micrometre can reproduce the observations. The inner disc radius is limited to $0.045 \mathrm{au}<R<0.52$ au within $1 \sigma$ confidence which corresponds to dust temperatures between 900 and $1750 \mathrm{~K}$. Besides amorphous carbon, the three other materials (amorphous carbonaceous analogues, crystalline graphite, astronomical silicate) show fitting results which are only slightly weaker $\left(\chi^{2}=2.325-2.387\right)$.

For the third and fourth approach (c and d), amorphous carbon is adopted as dust material. The best-fitting model of the third approach has a disc radius $R=0.1$ au, the dust temperature is $T_{\text {dust }} \sim 1430 \mathrm{~K}$, and the dust mass is $M_{\text {dust }}=2.0 \times 10^{-9} \mathrm{M}_{\oplus}$, and that of the fourth approach is $R=0.29 \mathrm{au}, T_{\text {dust }} \sim 940 \mathrm{~K}$, and $M_{\text {dust }}=$ $10.4 \times 10^{-9} \mathrm{M}_{\oplus}$. Within the $1 \sigma$ confidence level the dust location 
and temperature of both approaches are limited to $0.032-1.18$ au and $600-2000 \mathrm{~K}$. The grain size of both approaches amounts again to $a=0.58 \mu \mathrm{m}$ but is not constrained within the $1 \sigma$ confidence error. The dust properties and the SED of approach (b) are, as expected, the intermediate values of approach (c) and (d).

Finally, we have to verify that the disc model is compatible with the interferometric data. We take the maps of the best fits of the four approaches and compute their interferometric signal. The closure phases of all disc models are zero as a result of the face-on orientation. In Fig. 1, the computed visibilities of the disc model are shown as a function of baseline for approach (c). We can see that they approximate both the observational data and the visibilities of the uniform circumstellar emission. In particular, the visibility deficits of both circumstellar emission models (disc ring and Gaussian) compared to the pure stellar photosphere are similar. The main difference is the oscillations occurring for the disc model which are caused by the limited extension of the disc ring and which have been noticed also in the studies of Absil et al. (2009) and Kirchschlager et al. (2018). In summary, we conclude that the disc model is in line with the interferometric data.

\section{DISCUSSION AND CONCLUSIONS}

In this letter, we presented the first detection of hot exozodiacal dust emission in $L$ band. We used the new instrument MATISSE at the VLTI to observe the visibilities of $\kappa$ Tuc at six baselines. Using analytical solutions of a limb-darkened photosphere surrounded by uniform disc emission, we were able to derive significant $(>3 \sigma)$ dust-to-star flux ratios of 5-7 per cent in the wavelength range 3.37-3.85 $\mu \mathrm{m}$. Since the measured closure phases are close to zero, the MATISSE data strongly support the scenario that the excess is caused by circumstellar dust emission and not by a companion. The results present a further confirmation of the existence of hot exozodiacal dust around $\kappa$ tuc that has been detected previously (Ertel et al. 2014, 2016). In particular, the existence confirms that the temporal variability seen in previous PIONIER observations is caused by a variability of the dust properties. An explanation for the origin of the variability is beyond the scope of our study.

The newly derived $L$ band fluxes and the previously published $H$ band fluxes provided the basis for a SED modelling. The best fits were obtained for amorphous carbon grains of size $a=0.58 \mu \mathrm{m}$ though other carbons and astronomical silicate cannot be ruled out. Moreover, the grain size cannot be confined within the $1 \sigma$ confidence level. We note that the theoretical blow-out size of carbon grains around an F6 star amounts to $a_{\mathrm{BO}} \sim 1.4 \mu \mathrm{m}$ (Kirchschlager \& Wolf 2013) and smaller grains should be blown out of the system. However, this value neglects additional trapping mechanisms and has to be considered with caution (see e.g. Kral et al. 2017 for discussion).

Since the $H$ band data revealed a temporal variability, we combined them with the $L$ band data in different ways. Depending on the approach, the best fits are obtained for a narrow dust ring at a stellar distance in the $0.1-0.29$ au range, and thus with a temperature between 940 and $1430 \mathrm{~K}$ and total dust mass between $2 \times 10^{-9}$ and $10.4 \times 10^{-9} \mathrm{M}_{\oplus}$. Within the $1 \sigma$ confidence level dust location and temperature are constrained to $0.032-1.18$ au and $600-2000 \mathrm{~K}$.

The MATISSE observations open a new window to study hot exozodis. Though the $L$ band data alone can hardly determine the dust properties, the combination of $L$ band and NIR data $(H$ band) constrain the stellar distance of the emission. For a better understanding of the dust properties, and in particular for the location of the hot exozodi emission, simultaneous observations of $\kappa$ Tuc in the NIR and MIR domain are required in the future. The NIR flux has varied with a period of $\sim 12$ months between 2012 and 2014. Therefore, PIONIER and MATISSE observations conducted within a few months will allow to further constrain the dust location, and several sequences of these combined observations will help to understand the flux variability. Observations with VLTI/GRAVITY in $K$ band could support the study of the hot exozodi by filling the gap in the SED at an intermediate wavelength. The detection or nondetection of dust emission in $N$ band using MATISSE observations of higher quality can potentially determine or rule out the presence of silicate material due to the prominent silicate feature at $\lambda \sim 10 \mu \mathrm{m}$. Finally, high spectral resolution observations by MATISSE can be used to trace $L M$ band features that are consistent with certain materials. We note that $\kappa$ Tuc has a declination of $\delta \approx-69^{\circ}$ which is too far south to be visible for CHARA/FLUOR ( $K$ band) or the Large Binocular Telescope Interferometer ( $N$ band).

We have proven in this study that MATISSE offers the required sensitivity and spatial resolution for the observation of hot exozodis. In the future, the $L$ band and upcoming $M$ band observations will allow to determine the properties of other hot exozodis. Given the dust-to-star flux ratio of up to 7 per cent in $L$ band is a frequent phenomenon, MATISSE will most likely allow the discovery of new hot exozodis.

\section{ACKNOWLEDGEMENTS}

This study is based on observations collected at the European Organisation for Astronomical Research in the Southern hemisphere under ESO ID 0103.C-0725(A). FK was supported by European Research Council Grant SNDUST ERC-2015-AdG-694520. SW and AVK acknowledge support by the $\langle 0$ :funding-source $3: \mathrm{href}=$ "http://dx.d oi.org/10.13039/501100001659" $\rangle \mathrm{DFG}\langle/ 0$ :funding-source $\rangle$ through grants WO 857/15-2 and KR 2164/15-2. This research has benefited from the help of SUV, the VLTI user support service of the JeanMarie Mariotti Center (http://www.jmmc.fr/suv.htm).

\section{DATA AVAILABILITY}

The raw data are publicly available at the ESO archive. The reduced data will be made publicly available on the Optical Interferometry DataBase (OIDB) one year after the release of the raw data.

\section{REFERENCES}

Absil O. et al., 2006, A\&A, 452, 237

Absil O. et al., 2008, A\&A, 487, 1041

Absil O., Mennesson B., Le Bouquin J.-B., Di Folco E., Kervella P., Augereau J.-C., 2009, ApJ, 704, 150

Absil O. et al., 2013, A\&A, 555, A104

Agol E., 2007, MNRAS, 374, 1271

Ammler-von Eiff M., Reiners A., 2012, A\&A, 542, A116

Beckwith S. V. W., 2008, ApJ, 684, 1404

Chelli A., Duvert G., Bourgès L., Mella G., Lafrasse S., Bonneau D., Chesneau O., 2016, A\&A, 589, A112

Claret A., Diaz-Cordoves J., Gimenez A., 1995, A\&AS, 114, 247

Defrère D. et al., 2011, A\&A, 534, A5

Defrère D. et al., 2012, A\&A, 546, L9

Di Folco E. et al., 2007, A\&A, 475, 243

Draine B. T., Lee H. M., 1984, ApJ, 285, 89

Ertel S., Wolf S., Metchev S., Schneider G., Carpenter J. M., Meyer M. R., Hillenbrand L. A., Silverstone M. D., 2011, A\&A, 533, A132

Ertel S. et al., 2014, A\&A, 570, A128

Ertel S. et al., 2016, A\&A, 595, A44

Ertel S., Absil O., Defrère D., Augereau J. C., Mennesson B., 2018a, Exp. Astron., 46, 401 
Ertel S. et al., 2018b, AJ, 155, 194

Ertel S. et al., 2020, AJ, 159, 177

Fuhrmann K., Chini R., Kaderhandt L., Chen Z., 2017, ApJ, 836, 139

Gaia Collaboration, 2018, A\&A, 616, A1

Hanbury Brown R., Davis J., Lake R. J. W., Thompson R. J., 1974, MNRAS, 167,475

Jäger C., Mutschke H., Henning T., 1998, A\&A, 332, 291

Kimura H., Kunitomo M., Suzuki T. K., Robrade J., Thebault P., Mitsuishi I., 2020, Planet. Space Sci., 183, 104581

Kirchschlager F., Wolf S., 2013, A\&A, 552, A54

Kirchschlager F., Wolf S., Krivov A. V., Mutschke H., Brunngräber R., 2017, MNRAS, 467, 1614

Kirchschlager F., Wolf S., Brunngräber R., Matter A., Krivov A. V., Labdon A., 2018, MNRAS, 473, 2633

Kirchschlager F., Bertrang G. H. M., Flock M., 2019, MNRAS, 488, 1211

Kral Q. et al., 2017, Astron. Rev., 13, 69

Laor A., Draine B. T., 1993, ApJ, 402, 441
Lebreton J. et al., 2013, A\&A, 555, A146

Lopez B. et al., 2014, The Messenger, 157, 5

Marion L. et al., 2014, A\&A, 570, A127

Marshall J. P. et al., 2016, ApJ, 825, 124

Mennesson B. et al., 2014, ApJ, 797, 119

Millan-Gabet R. et al., 2011, ApJ, 734, 67

Nuñez P. D. et al., 2017, A\&A, 608, A113

Pearce T. D., Krivov A. V., Booth M., 2020, MNRAS

Rieke G. H., Gáspár A., Ballering N. P., 2016, ApJ, 816, 50

Rouleau F., Martin P. G., 1991, ApJ, 377, 526

Tokovinin A., 2020, AJ, 159, 265

van Lieshout R., Dominik C., Kama M., Min M., 2014, A\&A, 571, A51

Weingartner J. C., Draine B. T., 2001, ApJ, 548, 296

Woillez J. et al., 2019, A\&A, 629, A41

This paper has been typeset from a $\mathrm{T}_{\mathrm{E}} \mathrm{X} / \mathrm{L} \mathrm{T}_{\mathrm{E}} \mathrm{X}$ file prepared by the author. 